\title{
纳米柱表面等离子体共振的调制及其红外光谱增强特性研究
}

\author{
吕江涛 \\ 杨琳娟 \\ 李志刚 \\ 魏永涛 \\ 张宝健 \\ 梁丽勤 \\ 王凤文 \\ 司光远* \\ (东北大学 信息科学与工程学院 沈阳 110819)
}

\begin{abstract}
摘要 采用电子束直写光刻和离子束刻蚀的方法，将具有不同周期的纳米金柱阵列制备于透明的石英祄底上. 通过对 样品的透射光谱进行采集和分析可知, 改变纳米柱阵列周期可以实现在红外波段对局域表面等离子共振的精密调节. 实验结果表明，在透射谱中共振谷的波长随着周期的增大而红移，且红移的距离可以通过控制纳米柱阵列的周期进行 调制. 理论模拟结果与实验结果在一定程度上相吻合. 此外, 还对不同高度的纳米柱阵列的透射谱进行比较和分析. 更进一步, 将制备的纳米银柱阵列和傅里叶变换光谱仪的 ATR 附件相耦合, 有效增强了葡萄糖溶液的吸收谱强度. 可 见周期结构的纳米柱阵列可以有效增大样品表面的近场场强, 在信号传感和检测等领域有着广泛的应用前景.
\end{abstract}

关键词 纳米柱; 表面等离子体共振; 调制; 傅里叶变换红外光谱仪; 光谱增强

\section{Resonance Tuning in Plasmonic Nanorods for Enhanced Infrared Spectrum Detecting}

\author{
Lv, Jiangtao Yang, Linjuan Li, Zhigang Wei, Yongtao Zhang, Baojian \\ Liang, Liqin Wang, Fengwen Si, Guangyuan* \\ (College of Information Science and Engineering, Northeastern University, Shenyang 110819)
}

\begin{abstract}
Surface plasmons are electromagnetic waves propagating along metallic-dielectric interfaces and they have drawn considerable attention in the past ten years since extraordinary optical transmission (EOT) phenomenon was first reported. They can take different forms, from propagating waves to localized electron oscillations. Owning to their peculiar optical properties and particular capability of manipulating light at sub-wavelength scales, a wide range of practical applications have been enabled. Since the near-field of electromagnetic waves can be enhanced dramatically using plasmonic structures with different designs, surface plasmon based waveguides are of special importance to develop sensors and detectors with ultra-high sensitivity and figure of merit. Research on surface plasmons covers a broad scope with potential applications ranging from waveguiding to sensing. But plasmon assisted infrared detectors are rarely reported especially the ones working under near infrared (NIR) range. Tuning of surface plasmons in the NIR range is critical and essential to develop nanophotonic devices like modulators and sensors. Recently, plasmon assisted devices have drawn increasing interest. In this work, we show the combination of plasmonic nanorod arrays with NIR spectrum detection. Using electron-beam lithography $(\mathrm{EBL})$ and ion milling techniques, gold $(\mathrm{Au})$ nanorod arrays with varying periodicities were fabricated on transparent quartz substrates. By collecting and investigating the transmittance spectra of nanorods, precise tuning of localized surface plasmon resonance (LSPR) in the infrared range can be realized by changing the periodicity of nanorods. Experimental results show that the resonant wavelength of LSPR dips redshifts with increasing array periodicity and the distance of shift can be accurately controlled. Simulations show qualitative agreement with experimental results. The influence of waveguide sidewall on device performance is also discussed in detail. In addition, optical properties of nanorods with different heights were compared and investigated. By combining silver $(\mathrm{Ag})$ nanorod structures with attenuated total reflection-Fourier transform infrared (ATR-FTIR) spectroscopy technique, the absorbance of glucose can be effectively enhanced. Therefore, periodical nanorod arrays can significantly enhance the near field intensity, enabling extensive applications in signal sensing and detecting.

Keywords nanorods; surface plasmon resonance; tuning; FTIR; spectrum enhancement
\end{abstract}

\section{1 引言}

近年来，表面等离子体技术发展十分迅速，其应用 领域已经向其他学科领域交叉渗透，涉及领域包括：纳
米光子学 ${ }^{[1 \sim 5]}$ 、表面拉曼增强 ${ }^{[6,7]}$ 、亚波长光学 ${ }^{[8 \sim 10]}$ 、生 物传感器 ${ }^{[11 ~ 13]}$ 、纳米捕获 ${ }^{[14]}$ 、纳米波导 ${ }^{[15,16]}$ 、超级透

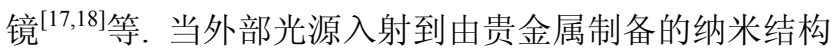
中时，会发生表面等离子体共振现象(surface plasmon

* E-mail: siguang0323@hotmail.com; Tel.: 13603239536

Received April 17, 2013; published June 13, 2013.

Project supported by the NEU Internal Funding (No. XNB201302), Natural Science Foundation of Hebei Province (No. A2013501049) and Fundamental Research Funds for the Central Universities (No. N120323014).

项目受东北大学校内博士启动基金(XNB201302)、河北省自然科学基金(A2013501049)和中央高校基本科研业务费(N120323014)资助. 
resonance, SPR). 这种共振是基于入射光与纳米结构之 间的相互作用和影响而形成的电子的振荡. 根据这种共 振是否能够沿着金属和介质的接触面传播可以将等离 子体共振分成局域型表面等离子体共振(localized surface plasmon resonance, LSPR)和传导型表面等离子共振 (propagating surface plasmon resonance, PSPR). 在连续 金属膜表面发生的共振, 称之为传导型等离子共振 (PSPR), 局限在纳米结构表面的共振则称之为局域型表 面等离子体共振(LSPR). 表面等离子体共振的强弱, 在很大程度上取决于所制备的纳米结构的形状. 随着纳 米材料制备技术的日趋成熟，可以制备得到各种各样的 贵金属纳米结构颗粒, 到目前为止设计的形状主要有环

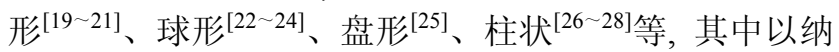
米柱的结构尤为重要. 在可见光和红外范围内, 纳米柱 表面等离子体结构是组成单层超材料以及超级透镜中 的一个重要的结构 ${ }^{[29 ~ 32]}$.

在纳米柱结构阵列中激发所产生的局域表面等离 子体共振, 大大增强了局域电磁场强度, 并且在不同的 结构中产生了强烈的近场耦合，这使得纳米结构具有了 较高的传感灵敏度和极大的品质因数以及优值系数. 这 种较高的传感灵敏度表现为高折射率, 其来自于纳米柱 表面等离子体和入射光之间的相互作用, 这种作用十分 稳定, 因此具有很强的可靠性. 在很多领域中所取得的 研究成果, 正是基于这种相互作用的稳定特性. 这种高 折射率的特性也使得局域表面等离子体共振现象获得 了广泛的应用. 局域表面等离子体共振的这种光学折射 率响应特性主要依赖于金属纳米结构的尺寸、形状、成 分和介质环境. 当我们需要将这种光学响应特性应用于 不同带宽的频率时, 便可以通过改变金属纳米阵列的尺 寸等参数特性, 进行不同纳米阵列光学结构的设计, 依 据弹性结构控制, 从而实现不同带宽的频率调制, 从而 使局域表面等离子体共振得到更加广泛的应用. 因此, 对于具有高品质因数和灵敏特性的局域表面等离子体 共振, 其可调性变得十分重要. 但是当纳米柱之间距离 接近到足以激起不同模式的等离子体之间的相互作用 的程度时, 金属纳米柱之间的耦合作用也会显著增强近 场的电磁场强度.

近年来, 金属纳米粒子表面等离子体的这种增强效 应在光谱领域得到了广泛的应用和发展. 例如, Kleinman 等 ${ }^{[33}$ 采用金纳米天线实现了拉曼光谱表面增强; Xie 等 ${ }^{[34]}$ 分析并验证了空心金纳米球的表面增强拉曼散 射效应; Hwang 等 ${ }^{[35]}$ 通过纳米金属表面等离子体共振技 术实现了荧光增强. 迄今为止, 采用金属表面等离子共 振技术进行光谱增强已经成为当前光谱增强领域研究 的热点. 但是从现有文献来看, 国内外对于纳米器件光 谱增强的研究, 主要集中在拉曼光谱和荧光光谱领域. 并且, 在红外范围内大部分的局域表面等离子体共振的 实验研究是通过使用周期等离子体晶体 (plasmonic crystal)结构来实现的. 因此，本文中为实现局域表面等 离子体共振在红外波长范围内的应用，通过改变纳米柱 阵列的周期，进行了局域表面等离子共振的调制，取得 了较好的调制效果. 本工作采用电子束直写光刻和离子 束刻蚀的方法进行了二维表面等离子体纳米柱结构的 高质量制备，详细介绍了制备过程，分析了纳米柱结构 的光学性质. 文中展示了在红外波段表面等离子体共振 的产生和对阵列周期的依赖性. 并将制备的金属纳米柱 阵列与傅里叶变换光谱仪(FTIR)的 ATR 附件相结合, 实现了葡萄糖溶液 ATR 光谱的显著增强. 这项研究在 等离子共振光谱增强领域将会具有广泛的应用.

\section{2 结果与讨论}

我们首先研究金 $(\mathrm{Au})$ 这种材料的相关特性. 图 1 为 所制备的纳米金柱阵列的扫描电子显微镜图(俯视图), 所示结构的周期为 $400 \mathrm{~nm}$, 插图为高倍数放大图.

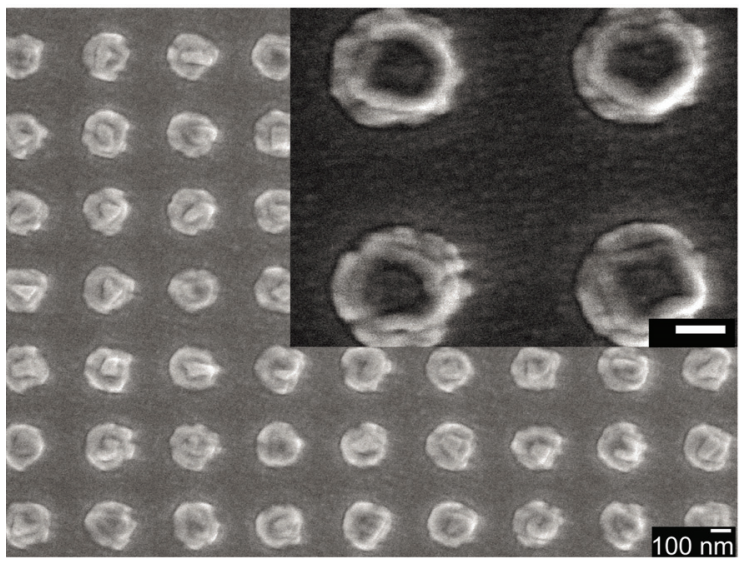

图 1 纳米柱阵列扫描电子显微镜俯视图(插图: 高倍放大图像, 插图 标尺: $100 \mathrm{~nm}$ )

Figure 1 SEM image showing the top view of a nanorod array Inset: high magnification image, scale bar: $100 \mathrm{~nm}$

从图中可以看出, 经过离子束刻蚀得到的纳米柱阵 列并不具备完美的圆柱形，这主要是由于制备方法本 身的局限性造成的. 这种非完美的纳米柱形状会在一定 程度上影响阵列的光学性能, 同时这也是造成测试结果 和理论模拟结果差异的主要原因. 此外, 模拟过程中金 属模型参数的选择以及边界条件的设置等也会导致实 验和模拟结果的不同. 在刻蚀过程中, 为了保证最好的 均一性, 样品随着固定样品用的圆盘一起以一定的速率 匀速转动，同时整个圆盘倾斜大约 $10^{\circ}$, 以使得更多的 离子经过电场加速后可以以较高速率从正入射的方向 轰击样品的表面, 从而得到均一性最佳的纳米柱阵列. 但是, 由于再沉积效应等因素的影响, 纳米柱的侧壁依 然是具有一定倾斜度的非垂直形状, 通常为穹顶状. 这 会导致入射光产生比较大的散射和吸收，从而影响透射 和反射等光学性能, 并会进一步影响作为传感和探测仪 
使用的相关器件的性能.

图 $2 \mathrm{a}$ 中所示为测试得到的具有不同周期的高度为 $60 \mathrm{~nm}$ 的金柱阵列的透射光谱. 可以看出, 局域表面等 离子共振谷在透射谱中随着周期的变化而发生位移. 周 期增大, 共振发生时的波长显著发生红移. 当周期为 $450 \mathrm{~nm}$ 时, 共振发生于 $1090 \mathrm{~nm}$ 左右, 周期减小为 400 $\mathrm{nm}$ 时, 共振谷移至 $990 \mathrm{~nm}$ 左右, 随着周期的进一步减 小, 共振发生时的波长也进一步蓝移. 在周期为 $200 \mathrm{~nm}$ 时, 共振波长达到约 $700 \mathrm{~nm}$. 此外, 从测试得到的透射 谱中还可以看出, 谱线具有相对较宽的半高全宽(Full Width at Half Maximum, FWHM), 比理论模拟得到的要 大. 在实际应用中, 为了提高传感器的精度可以使用折 射率和祄底一致的液体(如 $n=1.46$ 的油)覆盖样品表面, 使得纳米柱阵列上、下介质的折射率差异为 0 , 这样不 但可以减小半高全宽, 增大测试精度, 还能在最大限度 内将近场的增强效应提高. 可见, 通过精确控制阵列的 周期可以达到在红外波段调控共振波长的目的. 但是, 由于受制备方法和手段的限制，很难得到周期更小且致 密的纳米柱阵列, 因此想要把调制的范围进一步移至可 见光波段仍然是一个待解决的难题.
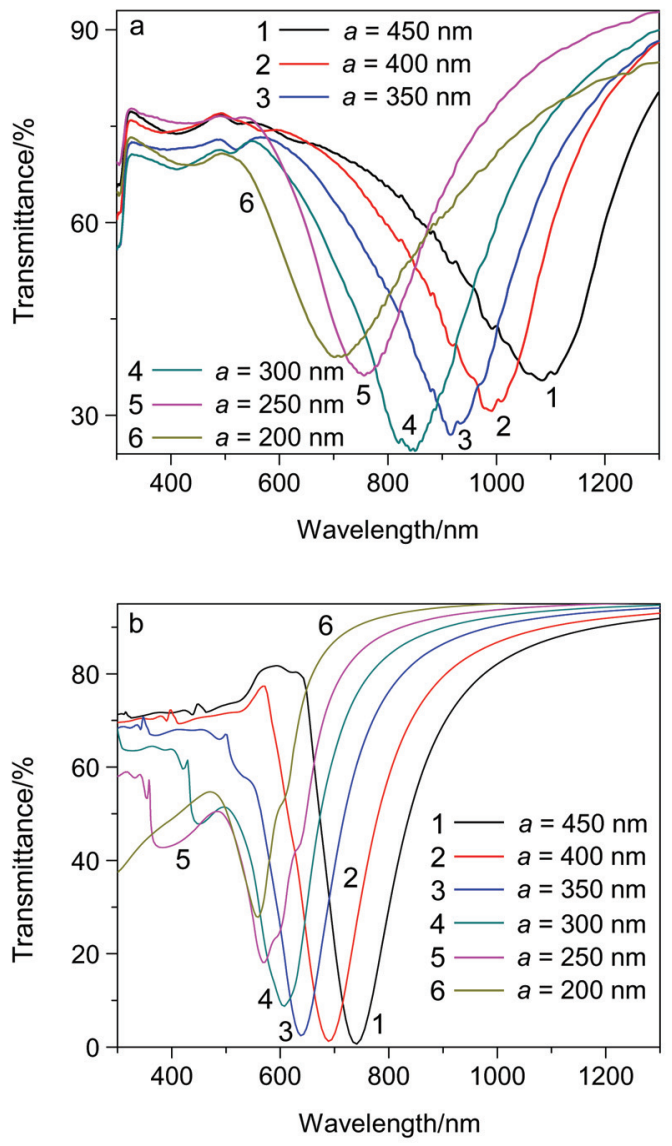

图 2 具有不同周期的纳米柱阵列的透射光谱 (a) 实验测得值; (b) 理论模拟值

Figure 2 The transmittance spectra of the nanorods array with different periodicities

(a) Experiments, (b) Simulations
从理论上分析，局域表面等离子共振可以用周期性 纳米结构的集合谐振理论来解释. 对于纳米柱阵列而 言，发生共振时的波长可以用公式(1) ${ }^{[36]}$ 来表示:

$$
\lambda_{R_{-} m p q}=\frac{a}{m}\left\{\sqrt{p^{2}+q^{2}} \pm \sin (\theta)[p \cos (\varphi)+q \sin (\varphi)]\right\}
$$

其中, $a$ 表示纳米柱阵列的周期, $\theta$ 是入射光入射到纳米 柱阵列上的入射角度, $\varphi$ 是入射光的偏振角度, $m, p, q$ 均 为整数. 对于正入射的情况(即 $\theta=0$ 时), 公式(1)可进一 步简化为

$$
\lambda_{R_{-} m p q}=\frac{a}{m} \sqrt{p^{2}+q^{2}}
$$

对于本工作中所关心的波段，最为重要的两个共振 波长是当 $p=1, q=0$ (或 $p=0, q=1$ ) 时所对应的共振波 长 $\lambda_{R_{-} m}^{\text {air }}$ 和 $p=q=1$ 时所对应的共振波长 $\lambda_{R_{-}{ }_{m}}^{\text {quarz }}$.

由公式(2)可知,

$$
\begin{aligned}
& \lambda_{R_{-} m}^{\text {air }}=\frac{a \cdot n_{\text {air }}}{m} \\
& \lambda_{R_{-} m}^{\text {quartz }}=\frac{a \cdot n_{\text {quartz }}}{m}
\end{aligned}
$$

这里, $n_{\text {air }}$ 为空气折射率, $n_{\text {quartz }}$ 为石英祄底折射率. 折射 率计算数值刚好与空气和所采用的石英基底的折射率 大小匹配，从而达到了共振模式.

处于阵列之中的纳米柱和单个的纳米柱在入射光 作用下受激发产生的表面等离子共振有很大不同. 阵列 中的相邻的纳米柱之间可以相互作用, 这种由相互作用 所产生的近场增强相较于单个的纳米柱而言会大很多. 这种增强技术可以应用到生物、化学传感，表面增强拉 曼散射，表面等离子体增强的显微成像等领域. 一般来 说，基于不同的柱间距(inter-rod spacing, 在图 6 中用 $s$ 表示)，有两种不同的耦合体系，分别叫做弱耦合体系 (weak coupling regime)和强耦合体系(strong coupling regime). 对于相对较大的柱间距, 远场偶极相互作用占 主导地位. 当柱间距明显地减小时，相邻的纳米柱能够 激发出很强的近场耦合．对于正方形结构的纳米柱阵 列，这种柱与柱之间的耦合作用可以用双极子(dipole) 模型 ${ }^{[37,38]}$ 来估计. 双极子模型可以很好地解释正方形的 纳米柱阵列所呈现的局域表面等离子体共振的谱线线 形，共振是在周期结构的衍射光栅和局限于纳米柱表面 的局域表面等离子体相互作用下形成的. 这种相互作用 受到阵列的周期布置的极大影响(最主要的则是周期的 大小).

图 $2 b$ 中所示为使用有限时域差分(finite-difference time-domain, FDTD)法理论模拟得到的不同周期的纳米 柱阵列的透射光谱. 共振波长随着纳米柱阵列的周期的 增加而红移的特性和实验结果相符. 当阵列周期为 450 $\mathrm{nm}$ 时，共振发生于 $720 \mathrm{~nm}$ 左右，周期减小到 $400 \mathrm{~nm}$ 时，共振波长蓝移至约 $690 \mathrm{~nm}$. 当阵列周期减小到 200 $\mathrm{nm}$ 时，共振波长最终蓝移至 $550 \mathrm{~nm}$. 理论计算中，对 
于同样阵列, 周期以 $50 \mathrm{~nm}$ 步长递增, 理论计算结果中 显示的共振波长的变化幅度比实验测得结果要小. 并且 对于相同周期的纳米柱阵列所对应的共振波长, 理论结 果普遍比实验测得结果偏小, 比如对于 $450 \mathrm{~nm}$ 的阵列, 理论模拟和实验测得的共振波长分别是 $730 \mathrm{~nm}$ 和 1090 $\mathrm{nm}$. 此外, 理论模拟谱线中的共振谷的半高全宽也小于 实验测得结果.

为了更加形象和直观地比较实验和理论模拟结果 的差异, 我们用图 3 来说明共振发生时的波长与阵列周 期的关系. 造成理论和实验之间差异的最主要原因是由 于实验制备得到的具有结构缺陷(参差不齐的边界、由 再沉积效应造成的粗粘的器件表面和不垂直的侧壁)的 纳米柱阵列和理论模拟中所使用的完美模型(完全光滑 的表面和垂直的纳米柱侧壁)之间的不同造成的.

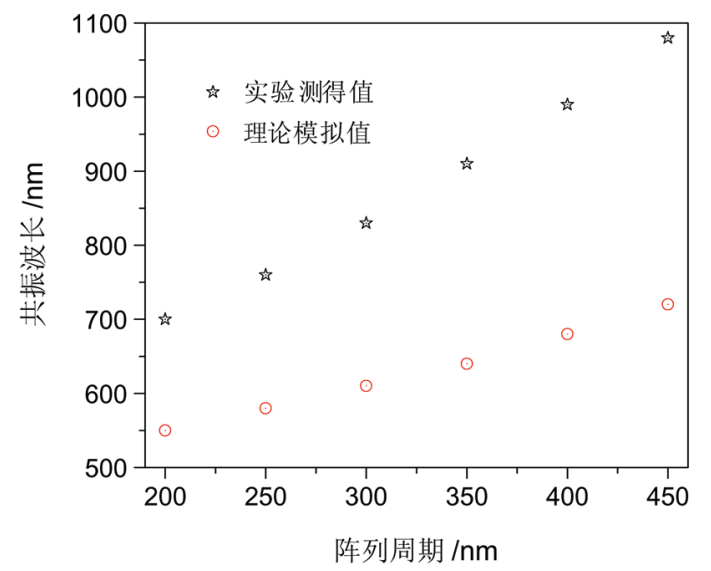

图 3 共振发生时的波长 $\left(\lambda_{\min }\right)$ 与纳米柱阵列周期的关系

Figure 3 The relationship between the LSPR wavelength and the array periodicity

为了更好的应用纳米柱阵列等离子体共振效应, 本 文进一步研究了纳米柱高度对其光学特性的影响. 我们 实验比较了 $60 \mathrm{~nm}$ 和 $300 \mathrm{~nm}$ 高的纳米柱阵列的透射谱, 其结果如图 4 所示.

可以看到, 由于纳米柱的显著增高, 对于周期为 $450 \mathrm{~nm}$ 高度为 $300 \mathrm{~nm}$ 的方形纳米柱阵列, 相比较于周 期为 $450 \mathrm{~nm}$ 高度为 $60 \mathrm{~nm}$ 的方形纳米柱阵列透射的能 量显著减少, 在 $400 \sim 1100 \mathrm{~nm}$ 波长范围内, $300 \mathrm{~nm}$ 高度 的纳米柱阵列强度普遍低于 $45 \%$. 和 $60 \mathrm{~nm}$ 高度的阵列 相比, 平均低了约 $30 \%$. 这主要是因为随着纳米柱高度 的增加, 更多的能量被阵列反射回并耦合到反射光中, 另有一部分能量由于一些模式限制在纳米柱之间的腔 体中而损耗消逝掉了. 这就造成了可以透过 $300 \mathrm{~nm}$ 厚 的阵列的能量大幅减少. 另外, 相较于 $60 \mathrm{~nm}$ 高的纳米 柱阵列, 可以观察到更多的共振峰或谷在 $300 \mathrm{~nm}$ 的谱 线中, 这是因为更高阶的极子被激发, 双极子模型 (dipole)并不足以完整地表示和刻画柱与柱之间的相互 作用. 在透射谷波长处, 透射的能量很少(大部分被反

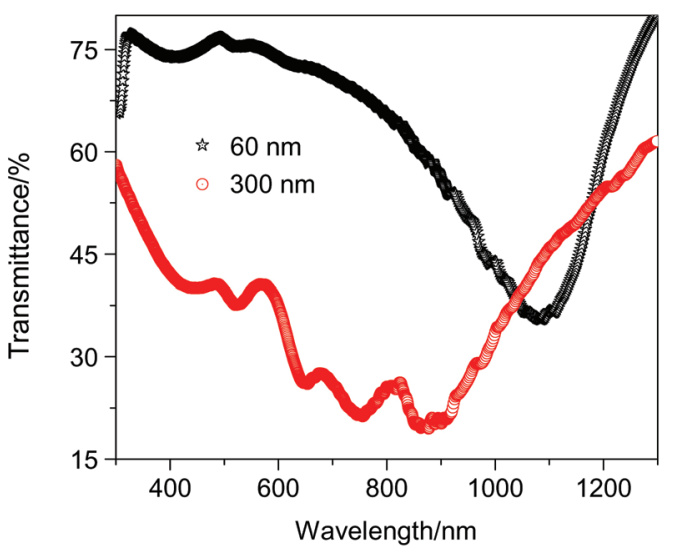

图 4 周期相同、高度分别为 60 和 $300 \mathrm{~nm}$ 的纳米柱阵列透射谱比较 Figure 4 The transmission spectrum of nanorod array with the same array periodicity and different heights

The height of the nanorods is $60 \mathrm{~nm}$ and $300 \mathrm{~nm}$, respectively

射回), 剩下的能量则主要局限于纳米柱的上表面和柱 与柱之间的腔体里.

为了展示相关结构的多样性和普遍适用性, 我们还 制备了高度为 $180 \mathrm{~nm}$ 的银 $(\mathrm{Ag})$ 纳米柱阵列(银相对于金 具有更高的离子刻蚀速率, 但是容易被氧化)并研究了 相关结构在光谱检测方面的可能应用. 图 5 中, 红色谱 线为 ATR 附件和银纳米柱相耦合后 $10 \%$ 的葡萄糖溶液 的吸收光谱，黑色谱线为 $10 \%$ 的葡萄糖溶液的 ATR 吸 收谱. 从图中我们可以看出, 在 3000 到 4000 波数范围 内, 水的吸收峰明显降低, 水的干扰得到了很好的抑制. 在 500 到 2000 波数范围内, 葡萄糖的吸收峰得到了显著 增强, 从而表明, 纳米柱阵列对于 FTIR-ATR 光谱有着 显著的增强效果.

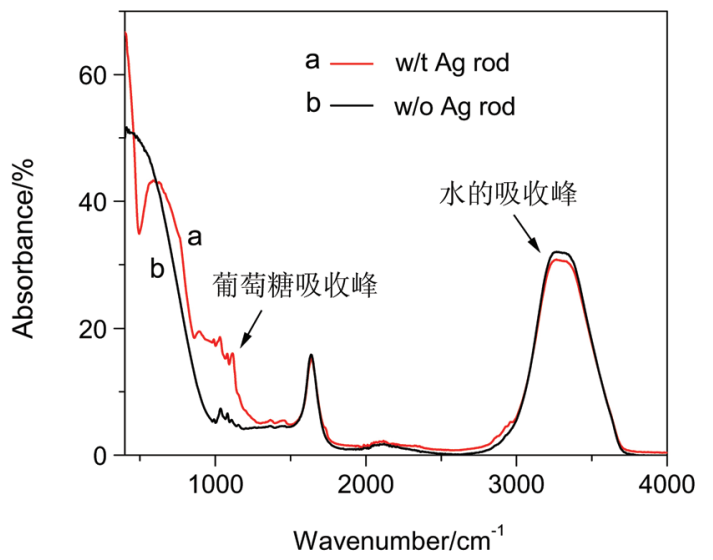

图 $510 \%$ 的葡萄糖溶液在覆盖银纳米柱 $(\mathrm{w} / \mathrm{t} \mathrm{Ag} \mathrm{rod})$ 和不覆盖纳米柱 (w/o Ag rod)时的 ATR 光谱对比

Figure 5 The ATR spectra of $10 \%$ glucose with and without Ag rods w/t: with; w/o: without

\section{3 结论}

本文实验展示了方形纳米柱阵列的制备，并将制备 的纳米柱阵列和 FTIR 的 ATR 附件相耦合, 实现了光谱 
的显著增强. 论文创新之处在于采用离子束刻蚀技术制 备了纳米柱阵列，通过控制阵列的周期实现了在红外波 段有效调节表面等离子体共振，并采用纳米柱阵列表面 等离子体共振效应，实现了 FTIR-ATR光谱的显著增强. 通过使用有限时域差分的方法, 将理论模拟结果和实验 测得的结果比较, 二者在一定程度上吻合, 使得实验得 到了理论上的解释和支持. 所制备得到的纳米柱阵列结 构, 在入射光激发下所产生的局域表面等离子共振对阵 列的周围环境异常敏感, 阵列上、下两侧介质的折射率 不同, 会影响共振峰或谷的线形以及谱线的半高全宽. 文章还研究了纳米柱阵列的高度对其光学特性的相应 影响. 最后将制备的纳米柱阵列和 ATR 附件相耦合, 针 对 $10 \%$ 的葡萄糖溶液验证了纳米柱阵列表面等离子体 共振效应的显著光谱增强效果.

\section{4 实验部分}

实验测试所用的石英祄底(折射率为 1.46)首先经过 丙酮的超声清洗, 并用去离子水(de-ionized water)洗掉 丙酮和氮气枪将石英祄底吹干. 随后使用电子束蒸镀 (electron-beam evaporation)的方法, 在石英衬底上制备 厚度为 $60 \mathrm{~nm}$ 的金 $(\mathrm{Au})$ 膜并使用 $3 \mathrm{~nm}$ 厚的铬 $(\mathrm{Cr})$ 膜作为 粘附层. 使用的制备仪器为 EDWARDS Auto 306 Turbo E-beam Evaporator, 蒸镀过程中样品所处的封闭腔保持 压强为 $5 \times 10^{-7} \mathrm{mtorr}$ 左右. 为了保证得到平滑的金属膜 表面以尽量降低粗糙的器件表面对所制备的纳米柱阵 列的光学性能的影响, 电子束蒸镀时金属膜的沉积速率 维持在 $0.05 \mathrm{~nm} / \mathrm{s}$ 以下, 对应的总沉积时间约为 $20 \mathrm{~min}$. 随后，使用电子束直写(Electron Beam Lithography, EBL) 来定义纳米柱形状(使用负性电子光刻胶 NEB 22). 最后使用离子束刻蚀(ion milling)的方法将纳米柱阵列 结构从电子胶中转移到金属中. 离子束刻蚀的仪器为美 国 Veeco 公司的 Microetch 1201 ion beam etch 系统. 刻 蚀过程中, 加速电压和电流分别为 $300 \mathrm{~V}$ 和 $200 \mathrm{~mA}$. 在 流速为 $30 \mathrm{sccm}$ 时金的刻蚀速率约为 $23 \mathrm{~nm} / \mathrm{min}$. 使用离 子束刻蚀方法将纳米柱阵列结构从电子胶中转移到金 属中，可以有效避免采用丙酮或其他溶液浸泡电子胶使 其从样品表面剥离(lift-off)的方法所造成的阵列结构不 完整的问题. 尤其对于致密的纳米柱阵列, 转移方法不 得当, 有可能造成结构部分联接的现象. 若使用超声处 理样品则又可能造成剥离的面积过大而损伤纳米柱结 构. 使用离子束刻蚀则可有效避免这些情况的发生, 得 到均一的阵列结构. 此外, 在离子束刻蚀过程中要注意 保持样品的温度不能过高, 以免电子胶被烧坏, 导致随 后使用移除剂(remover 1165)去除电子胶的时候无法清 洗干净. 本工作使用 CRAIC 公司的产品 QDI $2010^{\mathrm{TM}}$ 显 微分光光度计对所制备的纳米柱阵列进行测试. 此仪器 使用 $75 \mathrm{~W}$ 的氙灯作为宽带白光源, 对应光谱则由内置 的光谱仪测得. 透射光谱测试使用空白的石英衬底作为
参照. 更多关于测试细节的描述可以参考文献[19]. 所 制备的纳米金柱示意图如图 6 所示, $a$ 和 $s$ 分别表示阵列 的周期和柱间距.

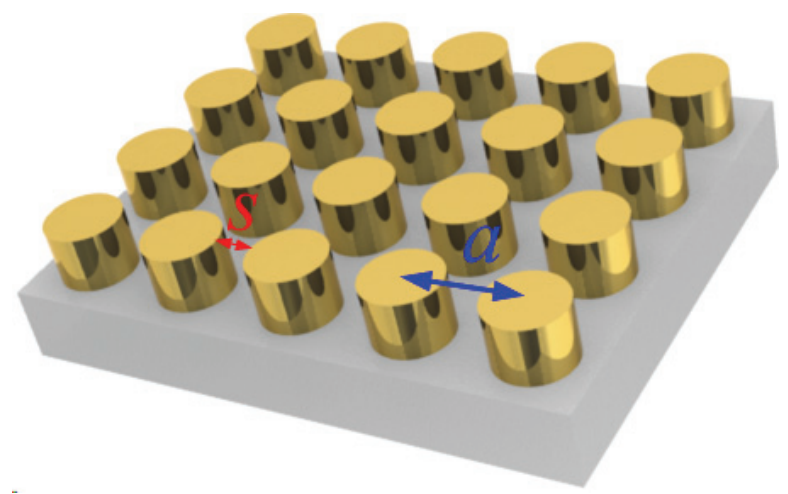

图 6 纳米金柱阵列制备于石英衬底上的示意图, 其中 $a$ 表示周期, $s$ 表示柱间距

Figure 6 Schematic of the gold nanorod array investigated in this work $a$ is the array periodicity, $s$ is the inter-rod spacing

图 7 所示为实验所用 FTIR 的 ATR 附件和银纳米柱 阵列相耦合测试的仪器示意图. 所用 ATR 附件为布鲁 克公司的 ALPHA 傅里叶变换红外光谱分析仪附件. 试 验中, 将 $10 \%$ 葡萄糖溶液样品填充于纳米柱阵列之间, 当入射光穿过 ATR 晶体入射到纳米柱阵列之间，便可 以引发金属纳米柱之间发生局部表面等离子体共振，这 样便大大增强传输波的能量，从而得到更加满意的吸收 谱，提高测量精度.

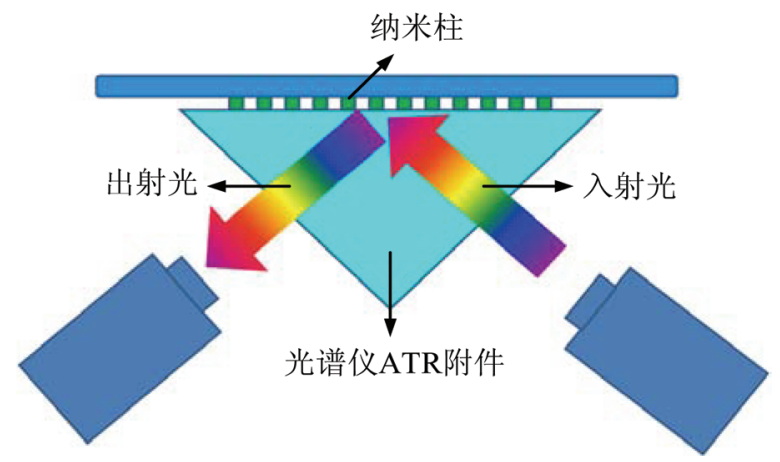

图 7 ATR 与银纳米柱阵列相耦合结构示意图

Figure 7 Schematic of the ATR structure combined with silver nanorod array for enhanced spectrum detection

\section{References}

[1] Wang, K.; Long, H.; Fu, M.; Yang, G.; Lu, P.-X. Opt. Lett. 2010, 35, 1560

[2] Cui, B.; Lin, H.; Zhao, X.-C.; Li, J.-B.; Li, W.-D. Acta Phys.-Chim. Sin. 2011, 27, 2411. (崔柏, 林红, 赵晓冲, 李建保, 李文迪, 物理 化学学报, 2011, 27, 2411.)

[3] Choi, J.-R. Chin. Phys. B 2010, 19, 010306.

[4] Todorov, Y.; Tosetto, L.; Teissier, J.; Andrews, A.-M.; Klang, P.; Colombelli, R.; Sagnes, I.; Strasser, G.; Sirtori, C. Opt. Express 2010, $18,13886$.

[5] Shen, L.-F.; Chen, X.-D.; Zhang, X.-F.; Agarwal, K. Plasmonics 2011, 6, 301 . 
[6] Zhang, Y.-Y.; Zhang, J. Acta Chim. Sinica 2012, 70, 2293. (张莹荣, 张锦, 化学学报, 2012, 70, 2293.)

[7] Fan, J.-F.; Zhao, C.-X.; Fan, L.-Z. Acta Chim. Sinica 2012, 70, 229. (范建凤, 赵晨醒, 范楼珍, 化学学报 2012, 70, 229.)

[8] Hibbins, A.-P.; Evans, B.-R.; Sambles, J.-R. Science 2005, 308, 670.

[9] Song, G.-F.; Wang, W.-M.; Cai, L.-K.; Guo, B.-S.; Wang, Q.; Xu, Y.; Wei, X.; Liu, Y.-T. Acta Phys. Sin. 2010, 59, 5105. (宋国峰, 汪卫 敏, 蔡利康, 郭宝山, 王青, 徐云, 韦欣, 刘运涛, 物理学报, 2010, 59, 5105.)

[10] Wang, Y.; Wang, X.; He, X.-J.; Mei, J. S.; Chen, M.-H.; Yin, J.-H.; Lei, Q.-Q. Acta Phys. Sin. 2012, 61, 137301. (王玥, 王暄, 贺训军, 梅金硕，陈明华，殷景华，雷清泉，物理学报, 2012，61，137301.)

[11] Li, Y.; Lin, Z.; Li, R.-Z.; Liu, X. Acta Chim. Sinica 2012, 70, 1304. (李迎, 林钢, 李蓉卓, 刘霞, 化学学报, 2012, 70, 1304.)

[12] Liu, X.; Sun, Y.; Song, D.-Q.; Tian, Y.; Zhang, H.-Q.; He, Y. Acta Chim. Sinica 2007, 65, 2544. (刘霞, 孙颖, 宋大千, 田媛, 张寒琦, 何彦, 化学学报, 2007, 65, 2544.)

[13] Wang, Q.; Zhu, H.-Z.; Yang, X.-H.; Wang, K.-M.; Yang, L.-J.; Ding, J. Acta Chim. Sinica 2012, 70, 1483. (王青, 朱红志, 羊小海, 王柯 敏, 杨丽娟, 丁静, 化学学报 2012, 70, 1483.)

[14] Quidant, R.; Girard, C. Laser \& Photon. Rev. 2008, 2, 47.

[15] Davoyan, A.-R.; Shadriivov, I.-V.; Kivshar, Y.-S. Opt. Express 2008, 16, 21209.

[16] Zhang, R.-J.; Qi, Z.-M.; Zhang, Z. Acta Phys.-Chim. Sin. 2011, 27, 1757. (张蓉君, 祁志美, 张喆, 物理化学学报, 2011, 27, 1757.)

[17] Zhang, Z.-Y.; Du, J.-L.; Guo, Y.-K.; Niu, X.-Y.; Li, M.; Luo, X.-G.; Du, C.-L. Chin. Phys. Lett. 2009, 26, 014211.

[18] Zhang, Z.-Y.; Du, J.-L.; Guo, X.-W.; Luo, X.-G.; Du, C.-L. J. Appl. Phys. 2007, 102, 074301.

[19] Lv, J.-T.; Wang, F.-W.; Ma, Z.-H.; Si, G.-Y. Acta Phys. Sin. 2013, 62, 057804. (吕江涛, 王凤文，马振鹤，司光远，物理学报，2013，62， 057804.)

[20] Jiang, X.-X.; Gu, Q.-C.; Wang, F.-W.; Lv, J.-T.; Ma, Z.-H.; Si, G.-Y. Mater. Lett. 2013, 100, 192.

[21] Si, G.-Y.; Zhao, Y.; Liu, H.; Teo, S.; Zhang, M.; Huang, T.-J.; Danner, A.-J.; Teng, J. Appl. Phys. Lett. 2011, 99, 033105.
[22] Hong, Q.-H.; Liu, X.-F.; Fang, Y. Acta Chim. Sinica 2013, 71, 255. (洪清华, 刘雪锋, 方云, 化学学报, 2013, 71, 255.)

[23] Tan, Y.; Ding, S.-H.; Wang, Y.; Qiang, W.-P. Acta Chim. Sinica 2005 , 63, 929 (谈勇, 丁少华, 王毅, 钱卫平, 化学学报, 2005, 63, 929.)

[24] Khoury, C.-G.; Norton, S.-J.; Vo-Dinh, T. ACS Nano 2009, 3, 2776.

[25] Guo, B.; Shan, W.-W.; Luo, J.-S.; Tang, Y.-J.; Cheng, J.-P. Acta Chim. Sinica 2008, 66, 1435 (郭斌，单雯雯，罗江山，唐永建，程 建平, 化学学报, 2008, 66, 1435.)

[26] Gao, Q.; Qian, Y.; Xia, Y.; Jiang, C.-Y.; Qiang, W.-P. Acta Chim. Sinica 2011, 69, 1617. (高倩, 钱勇, 夏炎, 蒋彩云, 钱卫平, 化学 学报, 2011, 69, 1617.)

[27] Si, G.-Y.; Zhao, Y.-H.; Lv, J.-H.; Wang, F.-H.; Liu, H.-L.; Teng, J.-H.; Liu, Y.-J. Nanoscale 2013, 5, 4309.

[28] Ke, S.-L.; Kan, C.-X.; Mo, B.; Cong, B.; Zhu, J.-J. Acta Phys.-Chim. Sin. 2012, 28, 1275. (柯善林，阚彩侠，莫博，从博，朱杰君，物理 化学学报, 2012, 28, 1275.)

[29] Yao, J.; Liu, Z.; Liu, Y.; Wang, Y.; Sun, C.; Bartal, G.; Stacy, A.-M.; Zhang, X. Science 2008, 321, 930 .

[30] Kabashin, A.-V.; Evans, P.; Pastkovsky, S.; Hendren, W.; Wurtz, G.-A.; Atkinson, R.; Pollard, R.; Podolskiy, V.-A.; Zayats, A.-V. Nat. Mater. 2009, 8, 867.

[31] Lemoult, F.; Fink, M.; Lerosey, G. Nat. Commun. 2012, 3, 889.

[32] Wurtz, G.-A.; Pollard, R.; Hendren, W.; Wiederrecht, G.-P.; Gosztola, D.-J.; Podolskiy, V.-A.; Zayats, A.-V. Nat. Nanotechnol. 2011, 6 , 107.

[33] Kleinman, S.-L.; Sharma, B.; Blaber, M.-G.; Henry, A.-I.; Valley, N.; Freeman, R.-G.; Natan, M.-J.; Schatz, G.-C.; Van Duyne, R.-P. J. Am. Chem. Soc. 2013, 135, 301.

[34] Xie, H.-N.; Larmour, I.-A.; Smith, W.-E.; Faulds, K.; Graham, D. J. Phys. Chem. C 2012, 116, 8338.

[35] Hwang, E.; Smolyaninov, I.-I.; Davis, C.-C. Nano Lett. 2010, 10, 813.

[36] Kravets, V.-G.; Schedin, F.; Grigorenko, A.-N. Phys. Rev. Lett. 2008, 101, 087403.

[37] Auguié, B.; Barnes, W.-L. Phys. Rev. Lett. 2008, 101, 143902.

[38] Atay, T.; Song, J. H.; Nurmikko, A.-V. Nano Lett. 2004, 4, 1627.

(Cheng, B.; Fan, Y.) 